\title{
Особенности спектров ИК отражения и комбинационного рассеяния кристаллов $\mathrm{Sb}_{2} \mathrm{Te}_{3-x} \mathrm{Se}_{x}$
}

\author{
(C) С.А. Немов ${ }^{1,2}$, В.Д. Андреева ${ }^{1}$, Ю.В. Улашкевич ${ }^{3}$, А.В. Поволоцкий ${ }^{4}$, А.А. Аллаххах ${ }^{1}$ \\ ${ }^{1}$ Санкт-Петербургский политехнический университет Петра Великого, \\ 195251 Санкт-Петербург, Россия \\ ${ }^{2}$ Санкт-Петербургский государственный электротехнический университет „ЛЭТИ“ им. В.И. Ульянова (Ленина), \\ 197376 Санкт-Петербург, Россия \\ ${ }^{3}$ Физико-технический институт им. А.Ф. Иоффре Российской академии наук, \\ 194021 Санкт-Петербург, Россия \\ ${ }^{4}$ Санкт-Петербургский государственный университет, \\ 199034 Санкт-Петербург, Россия \\ E-mail: nemov_s@mail.ru
}

(Получена 22 марта 2018 г. Принята к печати 28 марта 2018 г.)

\begin{abstract}
Проведено комплексное исследование (рентгенофазовое, комбинацион ного рассеяния и ИК отражения) кристаллов $p-\mathrm{Sb}_{2} \mathrm{Te}_{3-x} \mathrm{Se}_{x}(0 \leq x \leq 0.10)$, синтезированных методом Чохральского. Рентгенофазовый анализ и спектры комбинационного рассеяния света показали, что все исследованные образцы твердых растворов имеют кристаллическую ромбоэдрическую структуру с параметрами элементарной ячейки, близкими к их значениям в кристаллах $\mathrm{Sb}_{2} \mathrm{Te}_{3}$. Вместе с тем также наблюдается образование областей с разной степенью замещения атомов Те селеном. Спектры отражения исследованных кристаллов $R(v)$ имеют характерный минимум вблизи $1000 \mathrm{~cm}^{-1}$, который связывается с плазменными колебаниями дырок. Плазменный минимум закономерно смещается в высокочастотную сторону по мере увеличения концентрации селена. Расчет спектров отражения в рамках модели Друде-Лоренца удовлетворительно описывает экспериментальные спектры, причем в области низких частот необходимо учитывать также вклад оптических колебаний кристаллической решетки. Расчет позволил определить оптические параметры исследованных кристаллов, а также оценить эффективную массу дырок и оптическую проводимость.
\end{abstract}

DOI: 10.21883/FTP.2018.10.46461.8869

\section{1. Введение}

Неизменный интерес к тройным и четверным твердым растворам на основе халькогенидов $\mathrm{Sb}$ и $\mathrm{Bi}$ обусловлен тем, что в настоящее время они широко используются в термоэлектрических преобразователях энергии, работающих в диапазоне температур 200-500 K [1,2]. Дополнительный интерес к ним обусловлен возможностью существования в этих материалах состояния топологического изолятора [3-5], а также наблюдающимся расширением областей их применения. В частности, $\mathrm{Sb}_{2} \mathrm{Te}_{3}$ используется для изготовления омических контактов в солнечных элементах [6] и в ячейках памяти для информационных систем $[7,8]$.

Несмотря на то что интенсивные исследования этих соединений ведутся с середины прошлого века, полной ясности в электронных свойствах этих материалов, в их зонной структуре и механизмах рассеяния носителей тока до сих пор нет. Оптические свойства материалов $\mathrm{A}_{2}^{\mathrm{V}} \mathrm{B}_{3}^{\mathrm{VI}}$ также мало изучены [1]. Предварительные результаты исследования оптических свойств твердых раст воров $\mathrm{Sb}_{2} \mathrm{Te}_{3-x} \mathrm{Se}_{x}(x=0.05$ - образец 1 и $0.10-$ образец 2) в диапазоне $400-7000 \mathrm{~cm}^{-1}$ кратко изложены в статье [9]. В отличие от ранее исследованных кристаллов $\mathrm{PbSb}_{2} \mathrm{Te}_{4}$ и $\mathrm{Bi}_{2} \mathrm{Te}_{3}[10,11]$, в измеренных спектрах отражения наблюдался только один минимум (в районе $1000 \mathrm{~cm}^{-1}$ ), обусловленный плазменным резонансом дырок. Это согласуется с выводами, сделанными при более ранних исследованиях кристаллов $\mathrm{Sb}_{2} \mathrm{Te}_{3}$ [12], что позволило нам ограничить дальнейшие ИК измерения областью $4000 \mathrm{~cm}^{-1}$.

В настоящей работе выполнен рентгенофазовый анализ образцов 1 и 2, добавлены кристаллы с $x=0$ и 0.01, измерены спектры комбинационного рассеяния света (КРС) кристаллов твердых растворов $\mathrm{Sb}_{2} \mathrm{Te}_{3-x} \mathrm{Se}_{x}$ $(0 \leq x \leq 0.1)$ и продолжено исследование их оптических свойств в расширенном до $100 \mathrm{~cm}^{-1}$ спектральном диапазоне.

\section{2. Эксперимент}

Исследованные кристаллы были выращены методом Чохральского по технологии, разработанной в Институте металлургии и материаловедения им. А.А. Байкова РАН. Существенной особенностью технологии является использование плавающего графитового тигля специальной конструкции и подпитка жидким расплавом. Рост кристаллов осуществлялся в инертной атмосфере (гелий ОСЧ). Разработанная в ИМЕТ РАН технология обеспечивает получение кристаллов достаточно больших размеров в различных кристаллографических направлениях, в том числе вдоль тригональной оси $C_{3}(\sim 10 \mathrm{Mм})$, и с хорошей однородностью по химическому составу. Подробно технология описана в работе [13].

В средней инфракрасной области (MIR) спектры были измерены на спектрометре $1720 \mathrm{X}$ фирмы Perkin Elmer 


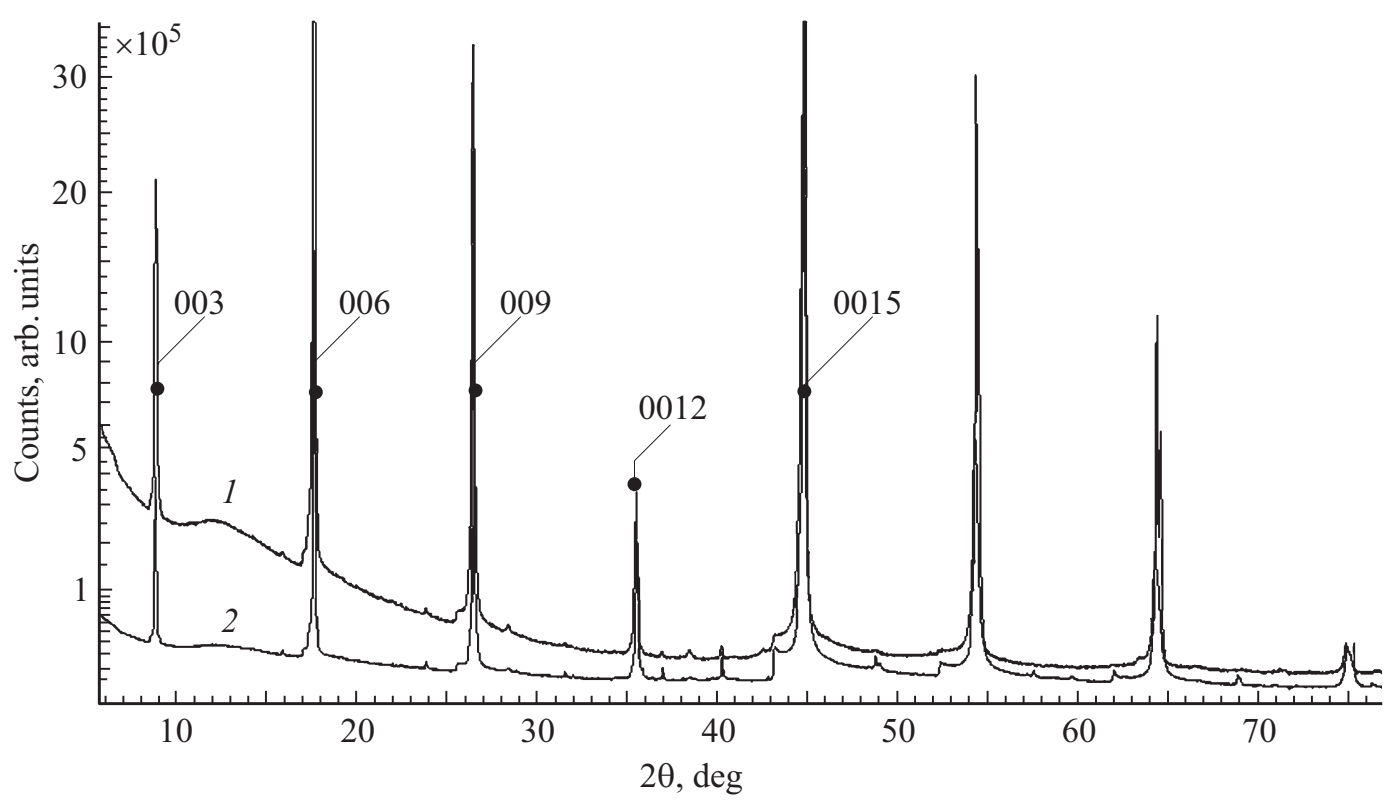

Рис. 1. Дифрактограммы кристаллов $\mathrm{Sb}_{2} \mathrm{Te}_{2.95} \mathrm{Se}_{0.05}$ (образец 1) и $\mathrm{Sb}_{2} \mathrm{Te}_{2.90} \mathrm{Se}_{0.10}$ (образец 2).

с помощью приставки отражения этой же фирмы. Для измерения в дальней инфракрасной области (FIR) использовался ИК фурье-спектрометр Nicolet 8700 фирмы Thermo Scientific, оснащенный приставкой для измерения спектров отражения и детектором: дейтерированный триглицинсульфат с полиэтиленовым окном (ДТГС/ПЭ). Спектральное разрешение приборов составляло $2 \mathrm{~cm}^{-1}$.

Для образцов проводилось несколько измерений, спектры усреднялись и „сшивались“ на общем краю диапазонов FIR и MIR. Каждое измерение проводилось со свежеприготовленным сколом кристалла по плоскости спайности за счет отрыва тонкого слоя кристалла с помощью скотча.

Рентгенофазовый анализ выполнен на дифрактометре D8 Advance с вертикальным гониометром $\theta-\theta$, фокусировкой по Бреггу-Брентано, на $\beta$-фильтрованном $\mathrm{Cu} K_{\alpha}$-излучении $(\lambda=1.5418 \AA$, Ni-фильтр), с вращением образца (30 об/мин) в плоскости держателя, регистрацией дифракционной картины высокоскоростным линейным детектором LynxEye фирмы Bruker (угол захвата $3.2^{\circ}$ ) в диапазоне углов $2 \theta=5-80^{\circ}$, с шагом сканирования $0.02^{\circ}$ и временем накопления $0.7 \mathrm{c} /$ шаг. Режимы работы трубки $U=40$ кВ, $I=40$ мА.

Для первичной обработки рентгенограмм (удаления фона) и определения фазового состава использовалось программное обеспечение DiffracPlus EVA (компания Bruker AXS) и порошковая база дифракционных данных ICDD PDF-2/Release 2008 [14].

Измерение спектров комбинационного рассеяния света (КРС) выполнялось с помощью спектрометра LabRam (Horiba) при возбуждении лазером с длиной волны генерации 632.8 нм, мощностью 3 мВт, в конфигурации обратного рассеяния с использованием конфокального микроскопа и микрообъектива с кратностью увеличения $\times 50$. Диаметр фокального пятна лазерного излучения на поверхности исследуемого образца можно было варьировать от 2 мкм. Каждый спектр измерялся 2 раза по 4 мин и усреднялся.

Измерение спектров с использованием оборудования Nicolet 8700 и LabRam проводилось в Ресурсном центре „Оптические и лазерные методы исследования вещества“ Научного парка СПбГУ.

\section{3. Результаты и их обсуждение}

Основные полученные экспериментальные данные приведены на рис. 1-5. Рассмотрение результатов начнем с данных по рентгенофазовому исследованию.

\section{1. Рентгенофазовое исследование кристаллов}

Информация о структуре кристаллов $\mathrm{Sb}_{2} \mathrm{Te}_{3-x} \mathrm{Se}_{x}$ c наибольшим содержанием селена получена рентгенофазовым исследованием поверхностей скола образцов 1 и 2. Зарегистрированные дифрактограммы представлены на рис. 1. Согласно положениям отражений от кристаллографических плоскостей $h k l$ можно считать, что основной фазой в исследованных образцах является соединение типа $\mathrm{Sb}_{2} \mathrm{Te}_{3-x} \mathrm{Se}_{x}$ (data_03-065-3678_PDF) [15] с пространственной группой $R \overline{\overline{3}} m$ и параметрами решетки $c=3.0407$ и 3.0369 нм в образцах 1 и 2 соответственно, при практически неизменном значении параметра $a=0.426$ нм. Расчет по методу Ритвельда в программе DiffracPlus Topas (фирма Bruker) [16] показал наличие незначительного количества аморфной фазы в образцах (проявляется на дифрактограммах в виде рентгеноаморфных гало). 
Таблица 1. Элементный состав образца 2

\begin{tabular}{c|c|c|c|c|c|c|c|c|c}
\hline $\begin{array}{c}\text { Спектр } \\
1\end{array}$ & Ат\% & Спектр & Ат\% & Спектр & Ат\% & Спектр & Ат\% & Спектр & Ат\% \\
\hline $\mathrm{Se}$ & 1.78 & $\mathrm{Se}$ & 1.87 & $\mathrm{Se}$ & 1.77 & $\mathrm{Se}$ & 1.83 & $\mathrm{Se}$ & 1.77 \\
$\mathrm{Sb}$ & 40.83 & $\mathrm{Sb}$ & 40.71 & $\mathrm{Sb}$ & 41.01 & $\mathrm{Sb}$ & 40.73 & $\mathrm{Sb}$ & 40.85 \\
$\mathrm{Te}$ & 57.38 & $\mathrm{Te}$ & 57.41 & $\mathrm{Te}$ & 57.22 & $\mathrm{Te}$ & 57.44 & $\mathrm{Te}$ & 5.38 \\
\hline Сумма & 100.00 & Сумма & 100.00 & Сумма & 100.00 & Сумма & 100.00 & Сумма & 100.00 \\
\hline \multicolumn{2}{c|}{$\mathrm{Sb}_{2} \mathrm{Te}_{2.80} \mathrm{Se}_{0.090}$} & \multicolumn{2}{|c|}{$\mathrm{Sb}_{2} \mathrm{Te}_{2.82} \mathrm{Se}_{0.092}$} & \multicolumn{2}{|c|}{$\mathrm{Sb}_{2} \mathrm{Te}_{2.78} \mathrm{Se}_{0.085}$} & \multicolumn{2}{|c|}{$\mathrm{Sb}_{2} \mathrm{Te}_{2.82} \mathrm{Se}_{0.090}$} & \multicolumn{2}{|c|}{$\mathrm{Sb}_{2} \mathrm{Te}_{2.80} \mathrm{Se}_{0.086}$}
\end{tabular}

Большая интенсивность дифракционных пиков 003, 006, 009 и т.д. основной фазы говорит о наличии ярко выраженной слоистости структуры, что позволяет рассматривать образцы как монокристаллические объекты. Анализ формы отдельных рефлексов образца 2 после выделения $K_{\alpha 1}$-составляющей (рис. 2) показал наличие на контурах дифракционных линий дополнительных диффузных максимумов. Это может быть связано с неоднородностью химического состава твердого раствора на различных участках поверхности кристалла. Известно, что $\mathrm{Se}$ способен замещать атомы теллура в $\mathrm{Sb}_{2} \mathrm{Te}_{3}$, уменьшая при этом параметр с решетки [1]. Это связано с замещением более крупного атома Те (размером 0.111 нм) меньшим по радиусу атомом $\mathrm{Se}(0.056 \mathrm{Hм})$. Постоянные решеток $c$ для $\mathrm{Sb}_{2} \mathrm{Te}_{3-x} \mathrm{Se}_{x}(0 \leq x \leq 1)$, согласно данным, представленным в структурной базе ICSD (Inorganic Crystal Structure Database) [17], меняются от 3.12 нм для $\mathrm{Sb}_{2} \mathrm{Te}_{3}$ $(x=0)$ (data_185946-ICSD) до 2.99 нм в соединении $\mathrm{Sb}_{2} \mathrm{Te}_{2} \mathrm{Se}(x=1)$ (data_2085-ICSD). Параметры решеток наблюдаемых нами твердых растворов укладываются в указанный диапазон и составляют от $c=2.9963$ нм до $c=3.0685$ нм. Таким образом, расщепление дифракционных максимумов на ряд диффузных пиков говорит о том, что в образцах наблюдаются химические ликвации с образованием областей с разной степенью замещения теллура атомами Se.

Энергодисперсионный анализ на микроанализатоpe OXFORD X-Max также показал (на примере $\mathrm{Sb}_{2} \mathrm{Te}_{2.9} \mathrm{Se}_{0.1}$ ), что количественный элементный состав в разных точках поверхности кристалла не одинаков и может соответствовать фазе с переменной стехиометрией $\mathrm{Sb}_{2} \mathrm{Te}_{3-x} \mathrm{Se}_{x}(0.085 \leq x \leq 0.092)$, что близко к исходному составу шихты (см. табл. 1$)$.

\section{2. Спектры комбинационного рассеяния}

Структурные особенности кристаллов исследовались с использованием спектров КРС. Как видно на рис. 3, в спектрах КРС всех образцов присутствуют интенсивные полосы в областях 69,113 и $163 \mathrm{~cm}^{-1}$, которые можно отнести к колебаниям $A_{1 g}^{1}, E_{g}^{2}$ и $A_{1 g}^{2}$ кристаллов $\mathrm{Sb}_{2} \mathrm{Te}_{3}[18,19]$. Таким образом, спектры КРС подтверждают вывод рентгенофазовых исследований, что основной кристаллической фазой для всех исследуемых образцов являются твердые растворы на основе $\mathrm{Sb}_{2} \mathrm{Te}_{3}$.

Увеличение концентрации селена в твердом растворе $\mathrm{Sb}_{2} \mathrm{Te}_{3-x} \mathrm{Se}_{x}$ приводит к появлению и росту КРС полос в

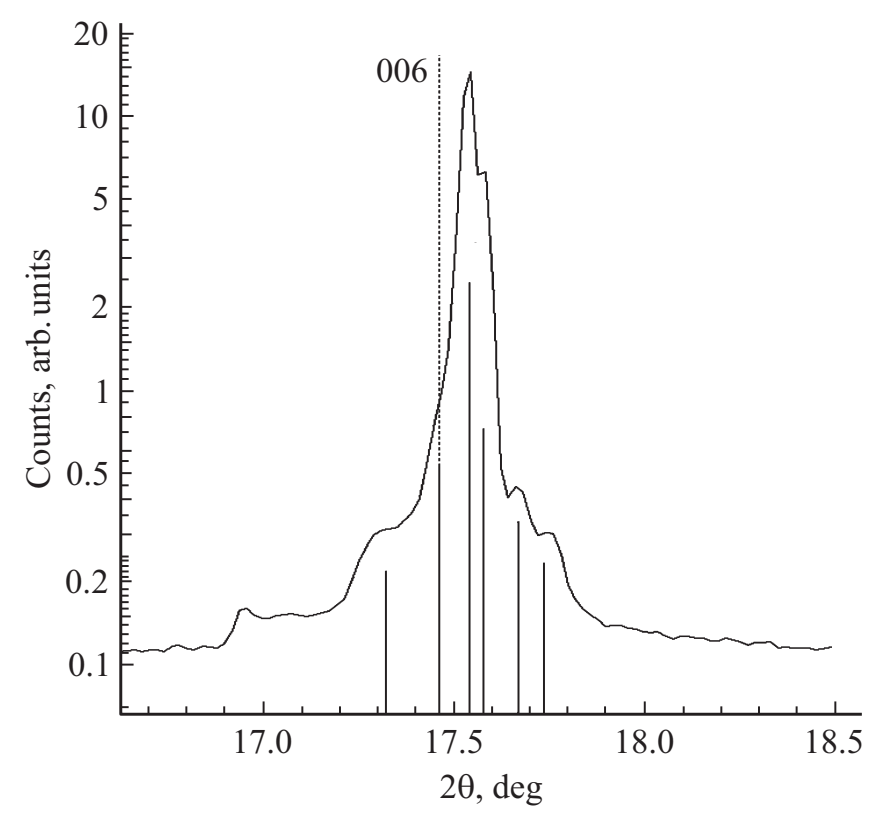

Рис. 2. Вид дифракционного пика 006 образца $\mathrm{Sb}_{2} \mathrm{Te}_{2.90} \mathrm{Se}_{0.10}$.

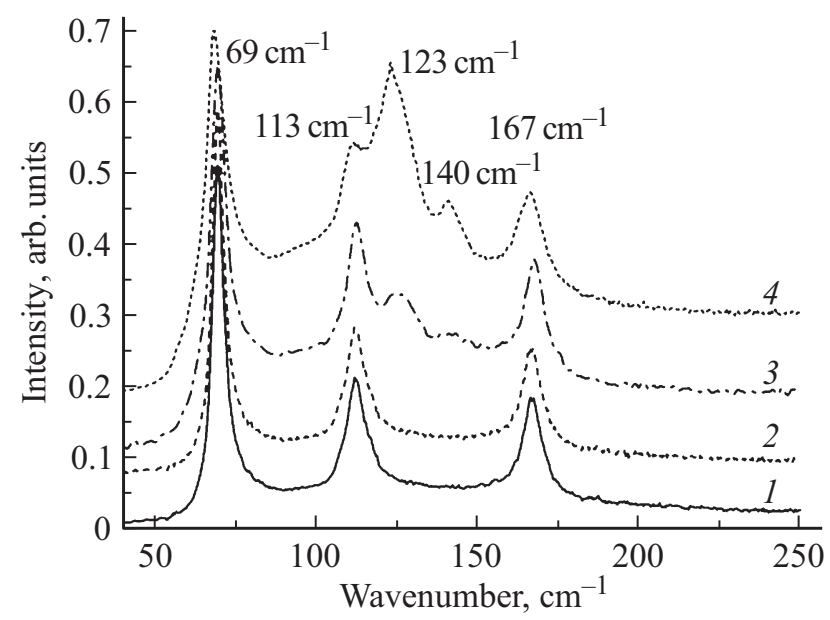

Рис. 3. Спектры КРС кристаллов $\mathrm{Sb}_{2} \mathrm{Te}_{3-x} \mathrm{Se}_{x}: x=0$ (1), 0.01 (2), 0.05 (3) и 0.10 (4). 
областях 123 и $140 \mathrm{~cm}^{-1}$. Полоса в области $123 \mathrm{~cm}^{-1}$, вероятно, связана с замещением Те атомами Se в твердом растворе $\mathrm{Sb}_{2} \mathrm{Te}_{3-x} \mathrm{Se}_{x}$, что приводит к сдвигу частоты колебаний $E_{g}^{2}$ в высокочастотную область. Полоса в области $140 \mathrm{~cm}^{-1}$ может быть обусловлена колебательными модами связи Те-Те [20] и не прореагировавшей $\mathrm{Sb}[21]$.

\section{3. Спектры инфракрасного отражения}

Как видно на рис. 4, спектры отражения $R(v)$ от сколов кристаллов $\mathrm{Sb}_{2} \mathrm{Te}_{3-x} \mathrm{Se}_{x}$ с содержанием селена $x=0,0.01,0.05$ и 0.10 подобны и имеют одинаковые характерные особенности, а именно присутствует

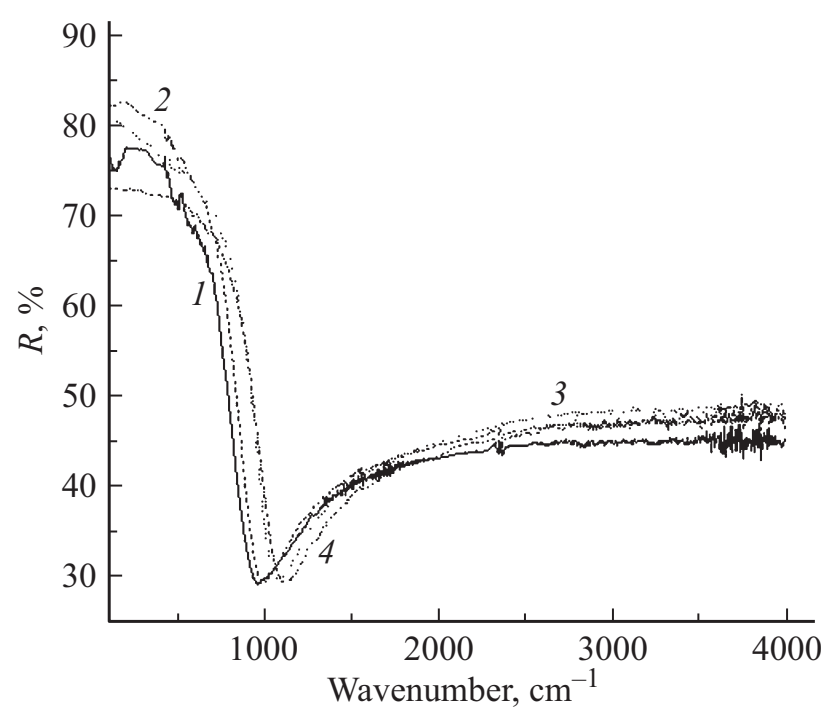

Рис. 4. Спектры отражения от сколов кристаллов $\mathrm{Sb}_{2} \mathrm{Te}_{3-x} \mathrm{Se}_{x}$ : $x=0$ (1), $0.01(2), 0.05$ (3) и $0.10(4)$.

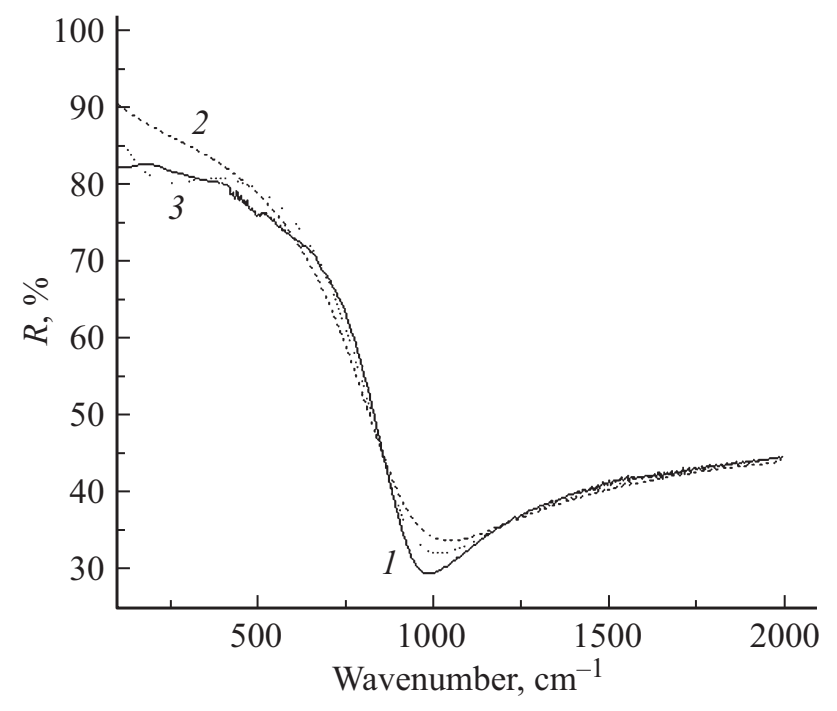

Рис. 5. Спектр отражения от скола кристалла $\mathrm{Sb}_{2} \mathrm{Te}_{2.99} \mathrm{Se}_{0.01}$ (1) и его аппроксимация согласно выражению (1) при $n=0$ (2) и $n=1$ (3). глубокий минимум в области $1000 \mathrm{~cm}^{-1}$. При больших частотах $\left(v>v_{\min }\right)$ наблюдается плавный подъем отражения с насыщением на уровне $50 \%$. В области более низких частот $\left(v<v_{\min }\right)$ наблюдается резкий подъем отражения также с тенденцией к насыщению на уровне (75-85)\%. Отмеченные особенности отражения полупроводников традиционно связываются с вкладом плазменных колебаний свободных носителей заряда (дырок в исследованных нами кристаллах) относительно ионов кристаллической решетки. Подобные особенности в ИК спектрах отражения наблюдаются в других материалах

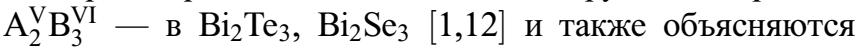
вкладом плазменных колебаний свободных носителей заряда в зависимость $R(v)$. Увеличение концентрации селена в кристаллах $\mathrm{Sb}_{2} \mathrm{Te}_{3-x} \mathrm{Se}_{x}$ приводит к закономерному смещению спектрального минимума в высокочастотную сторону: от $955 \mathrm{~cm}^{-1}$ при $x=0$ до $1115 \mathrm{~cm}^{-1}$ при $x=0.10$.

\section{4. Модель Друде-Лоренца}

Для описания оптических свойств проводящих материалов в рамках модели Друде-Лоренца используют диэлектрическую функцию, учитывающую вклад плазменных колебаний свободных носителей заряда и вклад колебаний $n$ осцилляторов в следующем общем виде [11]:

$$
\begin{aligned}
\varepsilon(v)= & \varepsilon_{1}(v)+i \cdot \varepsilon_{2}(v)=\varepsilon(\infty)+\frac{v_{p}^{2} \varepsilon_{\infty}}{-v^{2}+i v \Gamma} \\
& +\sum_{i}^{n} \frac{S_{i}}{v_{t i}^{2}-v^{2}+i v \gamma_{i}},
\end{aligned}
$$

где $\varepsilon_{1}$ и $\varepsilon_{2}-$ вещественная и мнимая составляющие диэлектрической проницаемости, $\varepsilon_{\infty}$ - высокочастотная (на оптических частотах) диэлектрическая проницаемость, $v_{t i}$ - частоты осцилляторных колебаний, $S_{i}$ - амплитуды осцилляторных колебаний, $\gamma_{i}-$ демпфирование осцилляторов, $v_{p}$ - плазменная частота $\left(\omega_{p}=2 \pi c v_{p}\right), \Gamma-$ демпфирование плазмы (время релаксации $\left.\tau_{p}=1 / 2 \pi c \Gamma\right), c-$ скорость света.

Результаты расчета аппроксимации согласно (1) с помощью нелинейного МНК представлены в табл. 2. Для сравнения с ранее приведенными данными в этой таблице в первой строчке приведен расчет, аналогичный [9]: $n=0$ в диапазоне $400-2500 \mathrm{~cm}^{-1}$. Следует отметить, что такая аппроксимация для спектров, измеренных от $100 \mathrm{~cm}^{-1}$, является неудовлетворительной, поскольку плохо описывает форму спектра вблизи плазменного минимума и завышает отражения в низкочастотной области (см. рис. 5). Поэтому в диэлектрической функции необходимо учитывать вклад колебаний кристаллической решетки, что и было сделано введением эффективных осцилляторов - третье слагаемое в формуле (1). Результаты такого расчета во всем исследованном диапазоне $100-4000 \mathrm{~cm}^{-1}$ при $n=1$ представлены в табл. 2. 
Таблица 2. Параметры аппроксимации спектров отражения кристаллов $\mathrm{Sb}_{2} \mathrm{Te}_{3-x} \mathrm{Se}_{x}$ моделью (1)

\begin{tabular}{c|c|c|c|c}
\hline $\begin{array}{c}\text { Параметры } \\
\text { кристаллов }\end{array}$ & 0.00 & 0.01 & 0.05 & 0.10 \\
\hline $\begin{array}{c}v_{p}, \mathrm{~cm}^{-1} \\
400-2500 \mathrm{~cm}^{-1}\end{array}$ & 878 & 915 & 998 & 1014 \\
$v_{p}, \mathrm{~cm}^{-1}$ & 361 & 545 & 614 & 439 \\
$100-4000 \mathrm{~cm}^{-1}$ & 27.0 & 30.4 & 32.3 & 29.6 \\
$\varepsilon_{\infty}$ & 1.26 & 0.71 & 0.57 & 1.08 \\
$m^{*} / m_{0}$ & 295 & 697 & 706 & 297 \\
$\sigma_{\mathrm{opt}}, \mathrm{M}^{-1} \cdot \mathrm{cM}^{-1}$ & & & &
\end{tabular}

Параметры $m^{*}$ и $\sigma_{\mathrm{opt}}$, приведенные в таблице, вычислялись согласно [11]. Здесь, однако, надо учитывать, что приведенные эффективные массы имеют завышенные значения, поскольку при их расчете использовались данные по концентрации дырок, определенные из коэффициентов Холла при азотной температуре. При комнатной же температуре в физических явлениях участвуют два типа дырок - легкие и тяжелые [22]. Поэтому эффективные массы $m^{*}$, вычисленные по однозонной формуле, являются завышенными.

\section{4. Заключение}

В заключение отметим, что рентгенофазовое исследование образцов 1 и 2 подтвердило, что основной фазой в них являются соединения типа $\mathrm{Sb}_{2} \mathrm{Te}_{3-x} \mathrm{Se}_{x}$ с пространственной группой $R \overline{3} m$ и характерными для этих соединений параметрами решетки. В то же время расщепление дифракционных максимумов на ряд диффузных пиков говорит о том, что в образцах наблюдаются химические ликвации с образованием областей с разной степенью замещения теллура атомами Se. Об этом же свидетельствует и энергодисперсионный анализ, выполненный для образца 2.

Спектры комбинационного рассеяния света в диапазоне $40-200 \mathrm{~cm}^{-1}$ были идентифицированы с использованием литературных данных и также подтверждают результаты рентгенофазового исследования кристаллов $\mathrm{Sb}_{2} \mathrm{Te}_{3-x} \mathrm{Se}_{x}$.

Спектры отражения исследованных кристаллов твердых растворов $\mathrm{Sb}_{2} \mathrm{Te}_{3-x} \mathrm{Se}_{x}(0 \leq x \leq 0.1)$ имеют особенности, характерные для плазменных колебаний свободных носителей. Плазменный минимум закономерно смещается в высокочастотную сторону с увеличением концентрации селена. Обсчет спектров отражения $R(v)$ в рамках модели Друде-Лоренца позволил определить параметры исследованных кристаллов, а также показал необходимость учета вклада колебаний кристаллической решетки в спектр отражения в области низких частот.

\section{Список литературы}

[1] Б.М. Гольцман, В.А. Кудинов, И.А. Смирнов. Полупроводниковые термоэлектрические материалы на основе $\mathrm{Bi}_{2} \mathrm{Te}_{3}$ (М., Наука, 1972).

[2] G.J. Snyder, E.S. Tobere. Nature Materials, 7, 105 (2008).

[3] Y.L. Chen, J.G. Analytis, J.-H. Chu, Z.K. Liu, S.-K. Mo, X.L. Qi, H.J. Zhang, D.H. Lu, X. Dai, Z. Fang, I.R.Zhang, S.C. Fisher, Z. Hussian, Z.-X. Shen. Science, 178, 5937 (2009).

[4] D. Hsieh, Y. Xa, D. Qian, L. Wray, F. Meier, J.H. Dil, J. Osterwalder, L. Patthey, A.V. Fedorov, H. Lin, A. Bansil, D. Grauer, Y.S. Hor, R.J. Cava, M.Z. Hasan. Phys. Rev. Lett., 103, 146401 (2009).

[5] С.В. Еремеев, Ю.М. Коротеев, Е.В. Чулков. Письма ЖЭТФ, 91, 419 (2010).

[6] G. Leimkuhler, I. Kerkamm, R. Reineke-Koch. J. Electrochem. Soc., 149, 474 (2002).

[7] M. Wuttig, M. Yamada. Nature Materials, 6, 824 (2007).

[8] C. Peng, Z.T. Song, F. Rao, L.C. Wu, M. Zhu, H. Song, B. Liu, X. Zhou, D. Yao, P. Yang, J. Chu. Appl. Phys. Lett., 99 (4), 043105 (2011).

[9] С.А. Немов, Ю.В. Улашкевич, А.А. Аллаххах, М.Б. Джафаров. ФТП, 51, 1034 (2017)

[10] С.А. Немов, Ю.В. Улашкевич, А.В. Поволоцкий, И.И. Хламов. ФТП, 50, 1343 (2016)

[11] С.А. Немов, Ю.В. Улашкевич, А.А. Аллахах. ФТП, 51, 1346 (2017).

[12] S.V. Dordevic, M.S. Wolf, N. Stojilovic, Hechang Lei, C. Petrovic. J. Phys.: Condens. Matter, 25, 075501 (2013).

[13] С.А. Немов, Г.Л. Тарантасов, В.И. Прошин, М.К. Житинская, Л.Д. Иванова, Ю.В. Гранаткина. ФТП, 43, 1629 (2009).

[14] DIFFRACplus Evaluation Package Release 2008-EVA V14 (GmbH, Karlsruhe, Germany).

[15] ICDD (The International Centre for Diffraction Data). http://www.icdd.com/

[16] DIFFRACplus TOPAS, Technical Reference. DOC-M88-EXX066, V4.2-01.2009(Bruker AXS GmbH, Karlsruhe, Germany).

[17] ICSD (Inorganic Crystal Structure Database). http://icsd.ill.fr/icsd/index.html

[18] W. Richter, H. Kohler, C.R. Becker. Phys. Status Solidi B, 84, 619 (1977).

[19] G.C. Sosso, S. Caravati, M. Bernasconi. J. Phys.: Condens. Matter, 21, 095410 (2009).

[20] A. Mendoza-Galván, E. García-García, Y.V. Vorobiev, J. González-Hernández. Microelectron. Engin., 51-52, 677 (2000).

[21] K.J. Tiwari, M.-Q. Ren, S.K. Vajandar, T. Osipowicz, A. Subrahmanyam, P. Malar. Solar Energy, 160, 56 (2018).

[22] С.А. Немов, Н.М. Благих, А.А. Аллаххах, Л.Д. Иванова, М.Б. Джафаров, А.Е. Демченко. ФТТ, 58, 2208 (2016)

Редактор Г.А. Оганесян 


\section{The features of the infrared reflection and Raman scattering spectra of $\mathrm{Sb}_{2} \mathrm{Te}_{3-x} \mathrm{Se}_{x}$ crystals}

S.A. Nemov ${ }^{1,2}$, V.D. Andreeva ${ }^{1}$, Yu.V. Ulashkevich ${ }^{3}$, A.V. Povolotsky ${ }^{4}$, A.A. Allahkhah ${ }^{1}$

${ }^{1}$ Peter the Great Polytechnical University, 195251 St. Petersburg, Russia

${ }^{2}$ St. Petersburg Electrotechnical University „LETl“, 197376 St. Petersburg, Russia

${ }^{3}$ loffe Institute,

194021 St. Petersburg, Russia

${ }^{4}$ St. Petersburg University, 199034 St. Petersburg, Russia

Abstract A complex research (the $X$-ray diffraction, the Raman scattering, and the infrared reflection) of $p-\mathrm{Sb}_{2} \mathrm{Te}_{3-x} \mathrm{Se}_{x}$ crystals $(0 \leq x \leq 0.10)$ synthesized by the Czochralski method was carried out. The $X$-ray phase analysis and Raman spectra showed that all the investigated samples of solid solutions had a crystalline rhombohedral structure with unit cell parameters close to their values in $\mathrm{Sb}_{2} \mathrm{Te}_{3}$ crystals. At the same time, formation of regions with different degrees of substitution of Te atoms with selenium is also observed. The reflection spectra of the investigated crystals $R(v)$ have a characteristic minimum near $1000 \mathrm{~cm}^{-1}$, which is associated with plasma oscillations of the holes. The plasma minimum naturally shifts to the high-frequency side as the concentration of selenium increases. The calculation of the reflection spectra within the framework of the Drude-Lorentz model satisfactorily describes the experimental spectra, and in the low-frequency region it is also necessary to take into account the contribution of the optical vibrations of the crystal lattice. The calculation made it possible to determine the optical parameters of the investigated crystals, as well as to estimate the effective mass of holes and optical conductivity. 